\title{
Energy Decay for a Von Karman Equation of Memory Type with a Delay Term*
}

\author{
Sun-Hye Park¹, Jong-Yeoul Park², Yong-Han Kang3\# \\ ${ }^{1}$ Center for Education Accreditation, Pusan National University, Busan, South Korea \\ ${ }^{2}$ Department of Mathematics, Pusan National University, Busan, South Korea \\ ${ }^{3}$ Institute of Liberal Education, Catholic University of Daegu, Gyeongsan, South Korea \\ Email: sh-park@pusan.ac.kr, jyepark@pusan.ac.kr, "yonghann@cu.ac.kr
}

How to cite this paper: Park, S.-H., Park, J.-Y. and Kang, Y.-H. (2017) Energy Decay for a Von Karman Equation of Memory Type with a Delay Term. Journal of Applied Mathematics and Physics, 5, 1797-1807. https://doi.org/10.4236/jamp.2017.59152

Received: August 9, 2017

Accepted: September 19, 2017

Published: September 22, 2017

Copyright $\odot 2017$ by authors and Scientific Research Publishing Inc. This work is licensed under the Creative Commons Attribution International License (CC BY 4.0).

http://creativecommons.org/licenses/by/4.0/

\begin{abstract}
We consider a von Karman equation of memory type with a delay term $\left|u_{t}\right|^{\rho} u_{t t}-\alpha \Delta u_{t t}+\Delta^{2} u-\int_{0}^{t} g(t-s) \Delta^{2} u(s) \mathrm{d} s+a_{0} u_{t}+a_{1} u_{t}(x, t-\tau)=[u, v]$. By introducing suitable energy and Lyapunov functional, we establish a general decay estimate for the energy, which depends on the behavior of $g$.

\section{Keywords}

Von Karman Equation, Memory Type, Delay Damping Term, General Decay Estimate
\end{abstract}

\section{Introduction}

Let $\Omega \subset \mathbb{R}^{2}$ be a bounded domain with sufficiently smooth boundary $\Gamma:=\partial \Omega$, $\Gamma_{0} \cup \Gamma_{1}=\Gamma, \quad \Gamma_{0} \cap \Gamma_{1} \neq \varnothing, \quad \Gamma_{0}$ and $\Gamma_{1}$ have positive measures and $v=\left(v_{1}, v_{2}\right)$ be the outward unit normal vector on $\partial \Omega$. We denote $u_{t}=\frac{\partial u}{\partial t}$, $\Delta u=\sum_{i=1}^{2} \frac{\partial^{2} u}{\partial x_{i}^{2}}$, where $x=\left(x_{1}, x_{2}\right) \in \Omega$.

In this paper, we investigate the decay of energy of solutions for a von Karman system with memory and a delay term

$$
\left\{\begin{array}{l}
\left|u_{t}\right|^{\rho} u_{t t}-\alpha \Delta u_{t t}+\Delta^{2} u-\int_{0}^{t} g(t-s) \Delta^{2} u(s) \mathrm{d} s+a_{0} u_{t}(x, t)+a_{1} u_{t}(x, t-\tau)=[u, v], \text { in } \Omega \times(0, \infty), \\
u=\frac{\partial u}{\partial v}=0 \text { on } \Gamma_{0} \times(0, \infty), \mathcal{B}_{1} u-\mathcal{B}_{1}\left(\int_{0}^{t} g(t-s) u(s) \mathrm{d} s\right)=0 \text { on } \Gamma_{1} \times(0, \infty), \\
\mathcal{B}_{2} u-\alpha \frac{\partial u_{t t}}{\partial v}-\mathcal{B}_{2}\left(\int_{0}^{t} g(t-s) u(s) \mathrm{d} s\right)=0 \text { on } \Gamma_{1} \times(0, \infty), \\
u(x, 0)=u_{0}(x), u_{t}(x, 0)=u_{1}(x), \quad x \in \Omega, u_{t}(x, t-\tau)=f_{0}(x, t-\tau), \quad(x, t) \in \Omega \times(0, \tau),
\end{array}\right.
$$

${ }^{*}$ This work was support by the national research foundation of Korea (Grant NRF2016R1D1A1B03930361).

\#Corresponding author. 
where $\rho$ is assumed to satisfy $0<\rho \leq \frac{2}{N-2}$ if $N \geq 3$ or $\rho>0$ if $N=1,2$, $\alpha>0 ; a_{0}$ is a positive constant; $a_{1}$ is a real number; $g$ is the kernel of the memory term; $\tau>0$ represents the time delay; $u_{0}, u_{1}, f_{0}$ are given functions belonging to suitable spaces; and the Airy stress function $v$ satisfies the following elliptic problem

$$
\left\{\begin{array}{l}
\Delta^{2} v=-[u, u] \text { in } \Omega \times(0, \infty), \\
\left.v=\frac{\partial}{\partial v} v=0 \text { on } \Gamma \times(0, \infty)\right) .
\end{array}\right.
$$

The von Karman bracket $[u, \phi]$ is given by

$$
[u, \phi] \equiv u_{x_{1} x_{1}} \phi_{x_{2} x_{2}}+u_{x_{2} x_{2}} \phi_{x_{1} x_{1}}-2 u_{x_{1} x_{2}} \phi_{x_{1} x_{2}},
$$

and

$$
\mathcal{B}_{1} u=\Delta u+(1-\mu) B_{1} u, \mathcal{B}_{2} u=\frac{\partial}{\partial v} \Delta u+(1-\mu) B_{2} u,
$$

here $\mu \in\left(0, \frac{1}{2}\right)$ is Poisson's ratio,

$$
\begin{gathered}
B_{1} u=2 v_{1} v_{2} u_{x_{1} x_{2}}-v_{1}^{2} u_{x_{2} x_{2}}-v_{2}^{2} u_{x_{1} x_{1}}, \\
B_{2} u=\frac{\partial}{\partial \tau}\left[\left(v_{1}^{2}-v_{2}^{2}\right) u_{x_{1} x_{2}}+v_{1} v_{2}\left(u_{x_{2} x_{2}}-u_{x_{1} x_{1}}\right)\right] .
\end{gathered}
$$

From the physical point of view, problem (1) describes small vibrations of a thin homogeneous isotropic plate of uniform thickness of $\alpha ; u=u(x, t)$ denotes the transversal displacement of the plate; the Airy stress function $v=v(x, t)$ is a vibrating plate.

When $a_{1}=0$ and $\rho=0$, problem (1) was studied by many authors [1]-[8]. The authors in [1] [3] [4] proved uniform decay rates for the von Karman system with frictional dissipative effects in the boundary. The stability for a von Karman system with memory and boundary memory conditions was treated in [5] [6] [7] [9]. They proved the exponential or polynomial decay rate when the relaxation function decay is at the same rate. The aim of this work is to prove a general decay result for a nonlinear von Karman equation of memory type with a delay term in the first equation of (1), when the relaxation function does not necessarily decay exponentially or polynomially. As for the works about general decay for viscoelastic system, we refer [10]-[15] and references therein. Considering delay term $a_{1} u_{t}(x, t-\tau)$, the problem is different from existing literature. Time delays arise in many applications depending not only on the present state but also on some past occurrences. And the presence of delay may be a source of instability (see e.g. [16] [17]). Thus, recently, the control of partial differential equations with time delay effects has become an active area of research (see [18] [17] [19] [20] and references therein). Nicaise and Pignotti [17] examined a wave equation with a time-delay of the form

$$
u_{t t}(x, t)-\Delta u(x, t)+a_{0} u_{t}(x, t)+a_{1} u_{t}(x, t-\tau)=0 .
$$


They proved that the energy of the problem decays exponentially under the condition

$$
0<a_{1}<a_{0}
$$

and there exists a sequence of delays such that instability occurs in the case $a_{1} \geq a_{0}$. Kirane and Said-Houari [21] considered a viscoelastic wave equation with a delay

$$
u_{t t}(x, t)-\Delta u(x, t)+\int_{0}^{t} g(t-s) \Delta u(x, s) \mathrm{d} s+a_{0} u_{t}(x, t)+a_{1} u_{t}(x, t-\tau)=0 .
$$

The authors proved the existence of a solution and a general decay result under the condition

$$
0<a_{1} \leq a_{0} .
$$

They showed that the energy of solutions is still asymptotically stable even if $a_{1}=a_{0}$ owing to the presence of the viscoelastic damping. Recently, $\mathrm{Wu}$ [20] obtained similar decay results as in [21] for problem (1) without von Karman bracket $[u, v]$ under the condition (5). Motivated by these results, we prove a general decay result for a nonlinear viscoelastic von Karman Equation (1) with a time-delay under the condition

$$
\left|a_{1}\right| \leq a_{0}
$$

which is an extension and improvement of the previous result from [20] to a nonlinear viscoelastic von Karman equation without the assumption $a_{1}>0$. The plan of this paper is as follows. In Section 2, we give some notations and materials needed for our work. In Section 3, we derive general decay estimate of the energy.

\section{Statement of Main Results}

Throughout this paper, we denote

$$
\begin{gathered}
V=\left\{u \in H^{3}(\Omega): u=0 \text { on } \Gamma_{0}\right\}, \\
W=\left\{u \in H^{2}(\Omega): u=\frac{\partial u}{\partial v}=0 \text { on } \Gamma_{0}\right\}, \\
(u, \phi)=\int_{\Omega} u(x) \phi(x) \mathrm{d} x \text { and }(u, \phi)_{\Gamma_{1}}=\int_{\Gamma_{1}} u(x) \phi(x) \mathrm{d} \Gamma .
\end{gathered}
$$

For a Banach space $X,\|\cdot\|_{X}$ denotes the norm of $X$. For simplicity, we denote $\|\cdot\|_{L^{2}(\Omega)}$ by $\|\cdot\|$ and $\|\cdot\|_{L^{2}\left(\Gamma_{1}\right)}$ by $\|\cdot\|_{\Gamma_{1}}$, respectively. We define for all $1 \leq p<\infty$

$$
\|u\|_{p}^{p}=\int_{\Omega}|u(x)|^{p} \mathrm{~d} x .
$$

From now on, we shall omit $x$ and $t$ in all functions of $x$ and $t$ if there is no ambiguity, and $c$ denotes a generic positive constant different from line to line or even in the same line.

For $0<\mu<\frac{1}{2}$, the bilinear form $a(\cdot, \cdot)$ is defined by

$a(u, \phi)=\int_{\Omega}\left\{u_{x_{1} x_{1}} \phi_{x_{1} x_{1}}+u_{x_{2} x_{2}} \phi_{x_{2} x_{2}}+\mu\left(u_{x_{1} x_{1}} \phi_{x_{2} x_{2}}+u_{x_{2} x_{2}} \phi_{x_{1} x_{1}}\right)+2(1-\mu) u_{x_{1} x_{2}} \phi_{x_{1} x_{2}}\right\} \mathrm{d} x .(7)$ 
A simple calculation, based on the integration by parts formula, yields

$$
\int_{\Omega} \Delta^{2} u \phi \mathrm{d} \Omega=a(u, \phi)-\left(\mathcal{B}_{1} u, \frac{\partial \phi}{\partial v}\right)_{\Gamma}+\left(\mathcal{B}_{2} u, \phi\right)_{\Gamma} .
$$

Thus, for $(u, \phi) \in\left(H^{4}(\Omega) \cap W\right) \times W$ it holds

$$
\int_{\Omega} \Delta^{2} u \phi \mathrm{d} \Omega=a\left((u, \phi)-\left(\mathcal{B}_{1} u, \frac{\partial \phi}{\partial v}\right)_{\Gamma_{1}}+\left(\mathcal{B}_{2} u, \phi\right)_{\Gamma_{1}} .\right.
$$

Since $\Gamma_{0} \neq \varnothing$ we know (see e.g. [1]) that $\sqrt{a(u, u)}$ is equivalent to the $H^{2}(\Omega)$ norm on $W$, i.e.

$$
c_{1}\|u\|_{H^{2}(\Omega)}^{2} \leq a(u, u) \leq c_{2}\|u\|_{H^{2}(\Omega)}^{2} \text { for some } c_{1}, c_{2}>0 .
$$

This and Sobolev imbedding theorem imply that for some positive constants $C_{p}, \tilde{C}_{p}$ and $C_{s}$

$$
\|u\|^{2} \leq C_{p} a(u, u),\|u\|_{\Gamma_{1}}^{2} \leq \tilde{C}_{p} a(u, u) \text { and }\|\nabla u\|^{2} \leq C_{s} a(u, u), \forall u \in W .
$$

By (7) and Young's inequality, we see that

$$
a(u, \phi) \leq \delta\|\phi\|_{H^{2}(\Omega)}^{2}+\frac{5}{8 \delta}\|\phi\|_{H^{2}(\Omega)}^{2} \text { for all } \delta>0 .
$$

From this and (8), it holds that

$$
a(u, \phi) \leq \delta a(u, u)+\frac{5}{8 c_{1}^{2} \delta} a(\phi, \phi) \text { for all } \delta>0 .
$$

We introduce the relative results of the Airy stress function and von Karman bracket $[\cdot, \cdot]$.

Lemma 2.1. ([4]) If $u, \phi$ and $\psi$ belong in $H^{2}(\Omega)$ and at least one of them belongs in $H_{0}^{2}(\Omega)$, then $([u, \phi], \psi)=([u, \psi], \phi)$.

Lemma 2.2. ([1]) Let $u \in H^{2}(\Omega)$ and $v$ be the Airy stress function satisfying (2). Then, the following relations hold:

$$
[u, v] \in L^{2}(\Omega) \text { and }\|[u, v]\| \leq C\|u\|_{H^{2}(\Omega)}\|v\|_{W^{2, \infty}(\Omega)} \leq \tilde{C}\|u\|_{H^{2}(\Omega)}\|u\|_{H^{2}(\Omega)}^{2} .
$$

Now, we state the assumptions for problem (1).

(H1) For the relaxation function $g$, as in [11] [15], we assume that $g: \mathbb{R}_{+} \rightarrow \mathbb{R}_{+}$is a nonincreasing differentiable function satisfying $g(0)>0$, $l_{0}:=\int_{0}^{\infty} g(s) \mathrm{d} s<\frac{1}{2}$ and

$$
g^{\prime}(t) \leq-\zeta(t) g(t) \text { for } t \geq 0,
$$

where $\zeta: \mathbb{R}_{+} \rightarrow \mathbb{R}_{+}$is a nonincreasing differentiable function.

Theorem 2.1. Assume that (H1) is hold. Then, for the initial data $\left(u_{0}, u_{1}, f_{0}\right) \in\left(H^{4}(\Omega) \cap W\right) \times\left(H^{3}(\Omega) \cap V\right) \times L^{2}(\Omega \times(0,1))$, problem (1) has a unique weak solution $u$ in the class

$$
u \in C\left(0, T ; H^{4}(\Omega) \cap W\right) \cap C^{1}\left(0, T ; H^{3}(\Omega) \cap V\right) .
$$

Proof. This can be proved by Faedo-Galerkin method (see e.g. [7] [21]). 


\section{General Decay of the Energy}

In this section we shall prove a general decay rate of the solution for problem (1). For simplicity of notations, we denote

$$
\begin{gathered}
g * u=\int_{0}^{t} g(t-s) u(s) \mathrm{d} s, \\
g \square u=\int_{0}^{t} g(t-s)\|u(t)-u(s)\|^{2} \mathrm{~d} s,
\end{gathered}
$$

and

$$
g \square \partial^{2} u=\int_{0}^{t} g(t-s) a(u(t)-u(s), u(t)-u(s)) \mathrm{d} s .
$$

From (9), we see that

$$
g \square u \leq C_{p} g \square \partial^{2} u .
$$

From now on, we shall omit $t$ in all functions of $t$ if there is no ambiguity, and $c$ denotes a generic positive constant different in various occurrences. Multiplying the first equation of (1) by $u_{t}$, we have

$$
E^{\prime}(t)=-a_{0}\left\|u_{t}\right\|^{2}-a_{1}\left(u_{t}(t-\tau), u_{t}\right)+a\left(g * u, u_{t}\right)
$$

where

$$
E(t)=\frac{1}{\rho+2}\left\|u_{t}\right\|_{\rho+2}^{\rho+2}+\frac{\alpha}{2}\left\|\nabla u_{t}\right\|^{2}+\frac{1}{2} a(u, u)+\frac{1}{4}\|\Delta v\|^{2} .
$$

From the symmetry of $a(\cdot, \cdot)$, we see that for any $u \in C^{1}\left(0, T ; H^{2}(\Omega)\right)$ $a\left(g * u, u_{t}\right)=-\frac{1}{2} g(t) a(u, u)+\frac{1}{2} g^{\prime} \square \partial^{2} u-\frac{1}{2} \frac{\mathrm{d}}{\mathrm{d} t}\left[g \square \partial^{2} u-\left(\int_{0}^{t} g(s) \mathrm{d} s\right) a(u, u)\right]$.

Moreover, (10) gives

$$
\begin{aligned}
a(g * u, u) & =\int_{0}^{t} g(t-s) a(u(s)-u(t), u(t)) \mathrm{d} s+\left(\int_{0}^{t} g(s) \mathrm{d} s\right) a(u, u) \\
& \leq 2\left(\int_{0}^{t} g(s) \mathrm{d} s\right) a(u, u)+\frac{5}{8 c_{1}^{2}} g \square \partial^{2} u .
\end{aligned}
$$

Now, we define a modified energy by

$$
\begin{aligned}
\mathcal{E}(t)= & \frac{1}{\rho+2}\left\|u_{t}\right\|_{\rho+2}^{\rho+2}+\frac{\alpha}{2}\left\|\nabla u_{t}\right\|^{2}+\frac{1}{2}\left(1-\int_{0}^{t} g(s) \mathrm{d} s\right) a(u, u) \\
& +\frac{1}{2} g \square \partial^{2} u+\frac{1}{4}\|\Delta v\|^{2}+\frac{p}{2} \int_{t-\tau}^{t}\left\|u_{t}(s)\right\|^{2} \mathrm{~d} s,
\end{aligned}
$$

where $p$ is a positive constant satisfying

$$
\left|a_{1}\right| \leq p \leq 2 a_{0}-\left|a_{1}\right| \text {. }
$$

It is noted that $E(t) \leq \frac{1}{1-l_{0}} \mathcal{E}(t)$. Therefore, it is enough to obtain the desired decay for the modified energy $\mathcal{E}(t)$ which will be done below.

Lemma 3.1. There exist non-negative constants $\alpha_{1}$ and $\alpha_{2}$ satisfying

$$
\mathcal{E}^{\prime}(t) \leq-\alpha_{1}\left\|u_{t}\right\|^{2}-\alpha_{2}\left\|u_{t}(t-\tau)\right\|^{2}-\frac{1}{2} g(t) a(u, u)+\frac{1}{2} g^{\prime} \square \partial^{2} u .
$$


Proof. Applying (14) to the last term in the right hand side of (13), we have

$$
\begin{aligned}
\mathcal{E}^{\prime}(t)= & -a_{0}\left\|u_{t}\right\|^{2}-a_{1}\left(u_{t}(t-\tau), u_{t}\right)-\frac{1}{2} g(t) a(u, u)+\frac{1}{2} g^{\prime} \square \partial^{2} u \\
& +\frac{p}{2}\left\|u_{t}\right\|^{2}-\frac{p}{2}\left\|u_{t}(t-\tau)\right\|^{2} .
\end{aligned}
$$

By Young's inequality,

$$
-a_{1}\left(u_{t}(t-\tau), u_{t}\right) \leq \frac{\left|a_{1}\right|}{2}\left\|\left.u_{t}\right|^{2}+\frac{\left|a_{1}\right|}{2}\right\| u_{t}(t-\tau) \|^{2} .
$$

Thus, we have

$$
\begin{aligned}
\mathcal{E}^{\prime}(t) \leq & -\left(a_{0}-\frac{p}{2}-\frac{\left|a_{1}\right|}{2}\right)\left\|u_{t}||^{2}-\left(\frac{p}{2}-\frac{\left|a_{1}\right|}{2}\right)\right\| u_{t}(t-\tau) \|^{2} \\
& -\frac{1}{2} g(t) a(u, u)+\frac{1}{2} g^{\prime} \square \partial^{2} u .
\end{aligned}
$$

Putting $\alpha_{1}=a_{0}-\frac{p}{2}-\frac{\left|a_{1}\right|}{2}, \alpha_{2}=\frac{p}{2}-\frac{\left|a_{1}\right|}{2}$ and considering (16), we complete the proof.

Now, let us define the perturbed modified energy by

$$
L(t)=N \mathcal{E}(t)+\varepsilon \Psi(t)+\theta \Upsilon(t)+\Phi(t),
$$

where

$$
\begin{gathered}
\Psi(t)=\frac{1}{\rho+1}\left(\left|u_{t}\right|^{\rho} u_{t}, u\right)+\alpha\left(\nabla u_{t}, \nabla u\right), \\
\Upsilon(t)=\int_{t-\tau}^{t} \mathrm{e}^{-(t-s)} \|\left. u_{t}(s)\right|^{2} \mathrm{~d} s, \\
\Phi(t)=-\frac{1}{\rho+1} \int_{0}^{t} g(t-s)\left(u(t)-u(s),\left|u_{t}\right|^{\rho} u_{t}\right) \mathrm{d} s \\
-\alpha \int_{0}^{t} g(t-s)\left(\nabla u(t)-\nabla u(s), \nabla u_{t}\right) \mathrm{d} s .
\end{gathered}
$$

Then, it is easily shown that $L(t)$ is equivalent with $\mathcal{E}(t)$ for all $t \geq 0$.

Lemma 3.2. There exist positive constants $C_{3}, C_{4}$ and $t_{0}>0$ satisfying

$$
\frac{\mathrm{d}}{\mathrm{d} t} L(t) \leq-C_{3} \mathcal{E}(t)+C_{4} g \square \partial^{2} u \text { for } t \geq t_{0} .
$$

Proof. Poincare's inequality gives

$$
\begin{aligned}
\Upsilon^{\prime}(t) & =-\int_{t-\tau}^{t} \mathrm{e}^{-(t-s)}\left\|u_{t}(s)\right\|^{2} \mathrm{~d} s+\left\|u_{t}(t)\right\|^{2}-\mathrm{e}^{-\tau}\left\|u_{t}(t-\tau)\right\|^{2} \\
& \leq-\mathrm{e}^{-\tau} \int_{t-\tau}^{t}\left\|u_{t}(s)\right\|^{2} \mathrm{~d} s+\lambda_{1}^{2}\left\|\nabla u_{t}(t)\right\|^{2}-\mathrm{e}^{-\tau}\left\|u_{t}(t-\tau)\right\|^{2},
\end{aligned}
$$

where $\lambda_{1}$ is the embedding constant from $H^{1}(\Omega)$ to $L^{2}(\Omega)$. Using the problem (1) and (14), we have

$$
\begin{aligned}
\Psi^{\prime}(t)= & \frac{1}{\rho+1}\left\|u_{t}\right\|_{\rho+2}^{\rho+2}+\alpha\left\|\nabla u_{t}\right\|^{2}+\alpha\left(\frac{\partial u_{t t}}{\partial v}, u\right)_{\Gamma_{1}}-a(u, u) \\
& +\left(\mathcal{B}_{1} u, \frac{\partial u}{\partial v}\right)_{\Gamma_{1}}-\left(\mathcal{B}_{2} u, u\right)_{\Gamma_{1}}+a(g * u, u)
\end{aligned}
$$




$$
\begin{aligned}
& -\int_{0}^{t} g(t-s)\left[\left(\mathcal{B}_{1} u(s), \frac{\partial u(t)}{\partial v}\right)_{\Gamma_{1}}-\left(\mathcal{B}_{2} u(s), u(t)\right)_{\Gamma_{1}}\right] \mathrm{d} s \\
& -a_{0}\left(u, u_{t}\right)-a_{1}\left(u_{t}(t-\tau), u\right)-\left(\Delta^{2} v, v\right) \\
= & \frac{1}{\rho+1}\left\|u_{t}\right\|_{\rho+2}^{\rho+2}+\alpha\left\|\nabla u_{t}\right\|^{2}-a(u, u)+a(g * u, u) \\
& -a_{0}\left(u, u_{t}\right)-a_{1}\left(u_{t}(t-\tau), u\right)-\|\Delta v\|^{2} \\
\leq & \frac{1}{\rho+1}\left\|u_{t}\right\|_{\rho+2}^{\rho+2}+\alpha\left\|\nabla u_{t}\right\|^{2}-\left(1-2 \int_{0}^{t} g(s) \mathrm{d} s\right) a(u, u) \\
& +\frac{5}{8 c_{1}^{2}} g \square \partial^{2} u-a_{0}\left(u, u_{t}\right)-a_{1}\left(u_{t}(t-\tau), u\right)-\|\Delta v\|^{2} .
\end{aligned}
$$

Young and Poincaré's inequalities produce

$$
\begin{gathered}
-a_{0}\left(u, u_{t}\right) \leq \eta a(u, u)+\frac{\lambda_{1}^{2} a_{0}^{2} C_{p}}{4 \eta}\left\|\nabla u_{t}\right\|^{2}, \\
-a_{1}\left(u_{t}(t-\tau), u\right) \leq \eta a(u, u)+\frac{a_{1}^{2} C_{p}}{4 \eta}\left\|u_{t}(t-\tau)\right\|^{2} .
\end{gathered}
$$

Substituting these into (21), we derive

$$
\begin{aligned}
\Psi^{\prime}(t) \leq & \frac{1}{\rho+1}\left\|u_{t}\right\|_{\rho+2}^{\rho+2}+\left(\alpha+\frac{\lambda_{1}^{2} a_{0}^{2} C_{p}}{4 \eta}\right)\left\|\nabla u_{t}\right\|^{2}-\left(1-2 l_{0}-2 \eta\right) a(u, u) \\
& +\frac{5}{8 c_{1}^{2}} g \square \partial^{2} u+\frac{a_{1}^{2} C_{p}}{4 \eta}\left\|u_{t}(t-\tau)\right\|^{2}-\|\Delta v\|^{2} .
\end{aligned}
$$

Similarly, we get from (1) that

$$
\begin{aligned}
\Phi^{\prime}(t)= & -\int_{0}^{t} g(t-s) \int_{0}^{t} g(t-\tau) a(u(\tau), u(t)-u(s)) \mathrm{d} \tau \mathrm{d} s \\
& +\int_{0}^{t} g(t-s) a(u(t), u(t)-u(s)) \mathrm{d} s-a_{0} \int_{0}^{t} g(t-s)\left(u_{t}(t), u(t)-u(s)\right) \mathrm{d} s \\
& -a_{1} \int_{0}^{t} g(t-s)\left(u_{t}(t-\tau(t)), u(t)-u(s)\right) \mathrm{d} s-\left([u, v], \int_{0}^{t} g(t-s)(u(t)-u(s)) \mathrm{d} s\right) \\
& -\frac{1}{\rho+1}\left(\left|u_{t}\right|^{\rho} u_{t}, \int_{0}^{t} g^{\prime}(t-s)(u(t)-u(s)) \mathrm{d} s\right)-\frac{1}{\rho+1} \int_{0}^{t} g(s) \mathrm{d} s\left\|u_{t}\right\|_{\rho+2}^{\rho+2} \\
& -\alpha\left(\nabla u_{t}, \int_{0}^{t} g^{\prime}(t-s)(\nabla u(t)-\nabla u(s)) \mathrm{d} s\right)-\alpha \int_{0}^{t} g(s) \mathrm{d} s\left\|\nabla u_{t}\right\|^{2} \\
:= & I_{1}+\cdots+I_{6}-\frac{1}{\rho+1} \int_{0}^{t} g(s) \mathrm{d} s\left\|u_{t}\right\|_{\rho+2}^{\rho+2}+I_{7}-\alpha \int_{0}^{t} g(s) \mathrm{d} s\left\|\nabla u_{t}\right\|^{2}
\end{aligned}
$$

In what follows we will estimate the terms in right hand side of (23). By similar arguments given in [8], we have

$$
\begin{gathered}
\left|I_{1}\right| \leq \delta l_{0}^{2} a(u, u)+\left(l_{0}+\frac{5 l_{0}}{8 c_{1}^{2}}+\frac{5 l_{0}}{8 c_{1}^{2} \delta}\right) g \square \partial^{2} u, \\
\left|I_{2}\right| \leq \delta l_{0} a(u, u)+\frac{5}{8 c_{1}^{2} \delta} g \square \partial^{2} u, \\
\left|I_{7}\right| \leq \delta \alpha\left\|\nabla u_{t}\right\|^{2}-\frac{\alpha g(0) C_{s}}{4 \delta}\left(g^{\prime} \square \partial^{2} u\right),
\end{gathered}
$$


and

$$
\begin{aligned}
\left|I_{5}\right| & \leq \delta\|[u, v]\|^{2}+\frac{1}{4 \delta}\left\|\int_{0}^{t} g(t-s)(u(t)-u(s)) \mathrm{d} s\right\|^{2} \\
& \leq \delta c\left(\|u\|_{H^{2}(\Omega)}\|u\|_{H^{2}(\Omega)}^{2}\right)^{2}+\frac{C_{p} l_{0}}{4 \delta} g \square \partial^{2} u \\
& \leq \tilde{c} \delta a(u, u)+\frac{C_{p} l_{0}}{4 \delta} g \square \partial^{2} u .
\end{aligned}
$$

Using Young inequality and the fact that imbedding $H^{1}(\Omega) \hookrightarrow L^{2(\rho+1)}$ is continuous, we infer

$$
\begin{aligned}
\left|I_{6}\right| & \leq \frac{1}{\rho+1}\left\{\delta\left\|u_{t}\right\|_{2(\rho+1)}^{2(\rho+1)}+\frac{1}{4 \delta}\left(\int_{0}^{t}-g^{\prime}(t-s)\|u(t)-u(s)\| \mathrm{d} s\right)^{2}\right\} \\
& \leq \frac{1}{\rho+1}\left\{\delta \lambda_{2}^{2(\rho+1)}\left\|\nabla u_{t}\right\|^{2(\rho+1)}+\frac{1}{4 \delta}\left(\int_{0}^{t} g^{\prime}(s) \mathrm{d} s\right) \int_{0}^{t} g^{\prime}(t-s)\|u-u(s)\|^{2} \mathrm{~d} s\right\} \\
& \leq \frac{\delta \lambda_{2}^{2(\rho+1)}}{\rho+1}\left(\frac{2 \mathcal{E}(0)}{\alpha}\right)^{\rho}\left\|\nabla u_{t}\right\|^{2}-\frac{g(0) C_{p}}{4 \delta(\rho+1)} g^{\prime} \square \partial^{2} u,
\end{aligned}
$$

where $\lambda_{2}$ is the embedding constant from $V$ to $L^{2(\rho+1)}(\Omega)$.

Young's inequality and (10) give

$$
\left|I_{3}\right| \leq \delta\left\|\nabla u_{t}\right\|^{2}+\frac{a_{0}^{2} l_{0} C_{p} \lambda_{1}^{2}}{4 \delta} g \square \partial^{2} u
$$

and

$$
\left|I_{4}\right| \leq \delta\left\|u_{t}(t-\tau)\right\|^{2}+\frac{a_{1}^{2} l_{0} C_{p}}{4 \delta} g \square \partial^{2} u
$$

Combining these estimates with (23), we get

$$
\begin{aligned}
\Phi^{\prime}(t) \leq & \delta\left(l_{0}+l_{0}^{2}+\tilde{c}\right) a(u, u)+\left\{\alpha\left(\delta-\int_{0}^{t} g(s) \mathrm{d} s\right)+\frac{\delta \lambda_{2}^{2(\rho+1)}\left(\frac{2 \mathcal{E}(0)}{\alpha}\right)^{\rho}}{\rho+1}+\delta\right\}\left\|\nabla u_{t}\right\|^{2} \\
& +\delta\left\|u_{t}(t-\tau)\right\|^{2}-\frac{1}{\rho+1}\left(\int_{0}^{t} g(s) \mathrm{d} s\right)\left\|u_{t}\right\|_{\rho+2}^{\rho+2}+c g \square \partial^{2} u-\frac{\alpha g(0) C_{s}+g(0) C_{p}}{4 \delta} g^{\prime} \square \partial^{2} u .
\end{aligned}
$$

Since $g$ is positive, for any $t_{0}>0$ we have $\int_{0}^{t} g(s) \mathrm{d} s \geq \int_{0}^{t_{0}} g(s) \mathrm{d} s:=g_{0}$ for all $t \geq t_{0}$. Thus, combining (17), (22) and (24), we arrive

$$
\begin{aligned}
\frac{\mathrm{d}}{\mathrm{d} t} L(t)= & N \mathcal{E}^{\prime}(t)+\epsilon \Psi^{\prime}(t)+\Phi^{\prime}(t) \\
\leq & -\left\{\alpha g_{0}-\delta\left(\alpha+1+\frac{\lambda_{2}^{2(\rho+1)}\left(\frac{2 \mathcal{E}(0)}{\alpha}\right)^{\rho}}{\rho+1}\right)-\epsilon\left(\alpha+\frac{\lambda_{1}^{2} C_{p} a_{0}^{2}}{4 \eta}\right)-\theta \lambda_{1}^{2}\right\}\left\|\nabla u_{t}\right\|^{2} \\
& -\frac{1}{\rho+1}\left(g_{0}-\epsilon\right)\left\|u_{t}\right\|_{\rho+2}^{\rho+2}-\left(\epsilon\left(1-2 l_{0}-2 \eta\right)-\delta\left(l_{0}+l_{0}^{2}+\tilde{c}\right)\right) a(u, u)
\end{aligned}
$$




$$
\begin{aligned}
& -\epsilon\|\Delta v\|^{2}-\left(\theta \mathrm{e}^{-\tau}-\frac{\epsilon a_{1}^{2} C_{p}}{4 \eta}-\delta\right)\left\|u_{t}(t-\tau)\right\|^{2}-\mathrm{e}^{-\tau} \int_{t-\tau}^{t}\left\|u_{t}(s)\right\|^{2} \mathrm{~d} s \\
& +\left[\frac{N}{2}-\frac{\alpha g(0) C_{s}+g(0) C_{p}}{4 \eta}\right] g^{\prime} \square \partial^{2} u+(c \epsilon+c) g \square \partial^{2} u .
\end{aligned}
$$

First, we fix $\eta>0$ and $\theta>0$ such that $1-2 l_{0}-2 \eta>0$ and $\alpha g_{0}-\theta \lambda_{1}^{2}>0$, respectively. Next, we choose $N>0$ so large that $\frac{N}{2}-\frac{\alpha g(0) C_{s}+g(0) C_{p}}{4 \eta}>0$, and $\epsilon>0$ sufficiently small such that $g_{0}-\epsilon>0$, $\alpha g_{0}-\theta \lambda_{1}^{2}-\epsilon\left(\alpha+\frac{\lambda_{1}^{2} C_{p} a_{0}^{2}}{4 \eta}\right)>0$ and $\theta \mathrm{e}^{-\tau}-\frac{\epsilon a_{1}^{2} C_{p}}{4 \eta}>0$. Finally, taking $\delta>0$ so small that $\alpha g_{0}-\theta \lambda_{1}^{2}-\epsilon\left(\alpha+\frac{\lambda_{1}^{2} C_{p} a_{0}^{2}}{4 \eta}\right)-\delta\left(\alpha+1+\frac{\lambda_{2}^{2(\rho+1)}\left(\frac{2 \mathcal{E}(0)}{\alpha}\right)^{\rho}}{\rho+1}\right)>0$, $\epsilon\left(1-2 l_{0}-2 \eta\right)-\delta\left(l_{0}+l_{0}^{2}+\tilde{c}\right)>0$ and $\theta \mathrm{e}^{-\tau}-\frac{\epsilon a_{1}^{2} C_{p}}{4 \eta}-\delta>0$, we complete the proof. $\square$

Theorem 3.1. There exist positive constants $C_{0}, \omega$ and $t_{0}>0$ such that

$$
E(t) \leq C_{0} \mathrm{e}^{-\omega \int_{t_{0}}^{t} \zeta(s) \mathrm{d} s} \text { for } t \geq t_{0}
$$

Proof. Multiplying (18) by $\zeta(t)$, using (11) and (17), we get

$$
\begin{aligned}
\zeta(t) L^{\prime}(t) & \leq-C_{3} \zeta(t) \mathcal{E}(t)+C_{4} \zeta(t) g \square \partial^{2} u \\
& \leq-C_{3} \zeta(t) \mathcal{E}(t)-C_{4} g^{\prime} \square \partial^{2} u \\
& \leq-C_{3} \zeta(t) \mathcal{E}(t)+C_{4}\left(-2 \mathcal{E}^{\prime}(t)\right) .
\end{aligned}
$$

Since $\zeta$ is nonincreasing, we have

$$
\left(\zeta(t) L(t)+2 C_{4} \mathcal{E}(t)\right)^{\prime} \leq-C_{3} \zeta(t) \mathcal{E}(t) \text { for } t \geq t_{0} .
$$

Thus, by letting $\mathcal{L}(t)=\zeta(t) L(t)+2 C_{4} \mathcal{E}(t)$, we get

$$
\mathcal{L}^{\prime}(t) \leq-c \zeta(t) \mathcal{E}(t) \text { for } t \geq t_{0} .
$$

Since $\zeta(t)$ is a nonincreasing positive function, we can easily observe that $\mathcal{L}(t)$ is equivalent to $\mathcal{E}(t)$. Subsequently, it follows that

$$
\mathcal{L}^{\prime}(t) \leq-c \zeta(t) \mathcal{E}(t) \text { for } t \geq t_{0} .
$$

Integrating this over $\left(t_{0}, t\right)$, we conclude that

$$
\mathcal{L}(t) \leq \mathcal{L}\left(t_{1}\right) \mathrm{e}^{-c \int_{t_{0}}^{t} \zeta(s) \mathrm{d} s} \text { for all } t \geq t_{0} .
$$

Consequently, the equivalent relations of $\mathcal{L}, L$ and $\mathcal{E}$ yield the desired result.

\section{Conclusion}

In this paper we proved decay rates of energy for a viscoelastic von Karman 
equation with constant time delay in the velocity by establishing proper Lyapunov functionals corresponding to the delay effect. In the future work, we will consider the equation with time-varying delay effect.

\section{References}

[1] Chueshov, I. and Lasiecka, I. (2004) Global Attractors for Von Karman Evolutions with a Nonlinear Boundary Dissipation. Journal of Differential Equations, 198, 196-231. https://doi.org/10.1016/j.jde.2003.08.008

[2] Chueshov, I. and Lasiecka, I. (2007) Long Time Dynamics of Von Karman Evolutions with Thermal Effects. Boletim da Sociedade Paranaense de Matemática, 25, 37-54.

[3] Favini, A., Horn, M.A., Lasiecka, I. and Tataru, D. (1996) Global Existence, Uniqueness and Regularity of Solutions to a Von Karman System with Nonlinear Boundary Dissipation. Differential Integral Equations, 9, 267-294.

[4] Lagnese, J.E. (1989) Boundary Stabilization of Thin Plates. SIAM, Philadelphia, PA. https://doi.org/10.1137/1.9781611970821

[5] Munoz Rivera, J.E. and Menzala, G.P. (1999) Decay Rates of Solutions of a Von Karman System for Viscoelastic Plates with Memory. Quarterly of Applied Mathematics, 57, 181-200. https://doi.org/10.1090/qam/1672191

[6] Munoz Rivera, J.E., Portillo Oquendo, H. and Santos, M.L. (2005) Asymptotic Behavior to a Von Karman Plate with Boundary Memory Conditions. Nonlinear Analysis, 62, 1183-1205. https://doi.org/10.1016/j.na.2005.04.025

[7] Park, J.Y. and Park, S.H. (2005) Uniform Decay for a Von Karman Plate Equation with a Boundary Memory Condition. Mathematical Methods in the Applied Sciences, 28, 2225-2240. https://doi.org/10.1002/mma.663

[8] Park, S.H., Park, J.Y. and Kang, Y.H. (2012) General Decay for a Von Karman Equation of Memory Type with Acoustic Boundary Conditions. Zeitschrift für Angewandte Mathematik und Physik, 63, 813-823. https://doi.org/10.1007/s00033-011-0188-2

[9] Lee, M.J., Park, J.Y. and Kang, Y.H. (2015) Exponential Decay Rate for a Quasilinear Von Karman Equation of Memory Type with Acoustic Boundary Conditions. Boundary Value Problems, 2015, 122. https://doi.org/10.1186/s13661-015-0381-x

[10] Cavalcanti, M.M., Domingos Cavalcanti, V.N. and Martinez, P. (2008) General Decay Rate Estimates for Viscoelastic Dissipative Systems. Nonlinear Analysis, 68, 177-193. https://doi.org/10.1016/j.na.2006.10.040

[11] Guesmia, A. and Messaoudi, S.A. (2009) General Energy Decay Estimates of Timoshenko Systems with Frictional versus Viscoelastic Damping. Mathematical Methods in the Applied Sciences, 32, 2102-2122. https://doi.org/10.1002/mma.1125

[12] Messaoudi, S.A. (2008) General Decay of Solutions of a Viscoelastic Equation. Journal of Mathematical Analysis and Applications, 341, 1457-1467.

[13] Messaoudi, S.A. and Mustafa, M.I. (2009) A Stability Result in a Memory-Type Timoshenko System. Dynamic Systems and Applications, 18, 457-468.

[14] Messaoudi, S.A. and Tatar, N.E. (2008) Exponential and Polynomial Decay for a Quasilinear Viscoelastic Equation. Nonlinear Analysis, 68, 785-793.

[15] Park, J.Y. and Park, S.H. (2009) General Decay for Quasilinear Viscoelastic Equations with Nonlinear Weak Damping. Journal of Mathematical Physics, 50, Article ID: 083505 . https://doi.org/10.1063/1.3187780 
[16] Datko, R. (1988) Not All Feedback Stabilized Hyperbolic Systems Are Robust with Respect to Small Time Delays in Their Feedbacks. SIAM Journal on Control and Optimization, 26, 697-713. https://doi.org/10.1137/0326040

[17] Nicaise, S. and Pignotti, C. (2006) Stability and Instability Results of the Wave Equation with a Delay Term in the Boundary or Internal Feedbacks. SIAM Journal on Control and Optimization, 45, 1561-1585. https://doi.org/10.1137/060648891

[18] Kafini, M., Messaoudi, S.A. and Mustafa, M.I. (2014) Energy Decay Rates for a Timoshenko-Type System of Thermoelasticity of Type III with Constant Delay. Applicable Analysis, 93, 1201-1216. https://doi.org/10.1080/00036811.2013.823480

[19] Suh, I.H. and Bien, Z. (1980) Use of Time Dealy Action in the Controller Desing. IEEE Transactions on Automatic Control, 25, 600-603. https://doi.org/10.1109/TAC.1980.1102347

[20] Wu, S.T. (2013) Asymptotic Behavior for a Viscoelastic Wave Equation with a Delay Term. Taiwanese Journal of Mathematics, 17, 765-784. https://doi.org/10.11650/tjm.17.2013.2517

[21] Kirane, M. and Said-Houari, B. (2011) Existence and Asymptotic Stability of a Viscoelastic Wave Equation with a Delay. Zeitschrift für angewandte Mathematik und Physik, 62, 1065-1082. https://doi.org/10.1007/s00033-011-0145-0

\section{Scientific Research Publishing}

Submit or recommend next manuscript to SCIRP and we will provide best service for you:

Accepting pre-submission inquiries through Email, Facebook, LinkedIn, Twitter, etc. A wide selection of journals (inclusive of 9 subjects, more than 200 journals)

Providing 24-hour high-quality service

User-friendly online submission system

Fair and swift peer-review system

Efficient typesetting and proofreading procedure

Display of the result of downloads and visits, as well as the number of cited articles

Maximum dissemination of your research work

Submit your manuscript at: http://papersubmission.scirp.org/

Or contact jamp@scirp.org 